\title{
Consumo televisivo de la población gitana y sus reflexiones sobre el reality show Los Gypsy Kings ${ }^{1}$
}

The televisual consumption by the Romani population and their reflexions about the reality show Los Gypsy Kings

Consumo televisivo da população cigana e suas reflexões sobre o reality show Los Gypsy Kings

DOI: https://doi.org/10.1590/1809-58442021301

\section{Gabriela Marques Gonçalves ${ }^{1}$}

https://orcid.org/0000-0002-9964-7757

${ }^{1}$ (Universitat Autónoma de Barcelona, Institut de la Comunicación. Barcelona - España).

\section{Resumen}

Este artículo tiene como objetivo discutir las percepciones de la población gitana de Barcelona (España) sobre el reality show español Los Gypsy Kings. Se hará una breve discusión teórica sobre la representación de la población gitana en los medios de comunicación españoles. Para comprender el debate, expondremos datos sobre su consumo televisivo, enfocando en los programas de entretenimiento, con informaciones sobre preferencias de cadenas de televisión y tipos de programas. Seguidamente, presentaremos las reflexiones de la muestra sobre el reality show. La metodología se basa en entrevistas semiestructuradas con 20 gitanos y gitanas realizadas entre los años 2017 y 2019. Los resultados señalan las semejanzas en la representación de las poblaciones gitanas en contenidos de entretenimiento e informativos. Vemos también que el consumo de reality shows y el debate público propuesto por asociaciones gitanas sobre Los Gypsy Kings están reflejados en las reflexiones de la muestra.

Palabras clave: Población Gitana. Consumo Televisivo. Representación. Los Gypsy Kings. Reality show.

\begin{abstract}
This paper aims to discuss the perceptions by the Romani population from Barcelona (Spain) about the Spanish reality show "Los Gypsy Kings". We will do a short theoretical discussion about the representation of the Romani population in Spanish media. To comprehend the debate, it will be showed data about their televisual consumption, focusing on entertainment programs, with some pieces of information about preferences related to television network broadcasts and types of programs. Next, it will be presented the reflections of the sample about the reality show
\end{abstract}

1 Investigación realizada mientras la autora era becaria de doctorado de Capes (MEC-Brasil). 
analyzed. The methodology consists of semi-structured interviews with 20 Romani women and men between 2017 and 2019. The results point out to the resemblance in the representation of the Romani populations in entertainment and informative contents. We also see that the consumption of reality shows and the public debate proposed by Romani associations about “Los Gypsy Kings” are reflected in the reflexions of the sample.

Keywords: Romani Population. Televisual Consumption. Representation. Los Gypsy Kings. Reality show.

\section{Resumo}

Este artigo tem como objetivo discutir as percepções da população cigana de Barcelona (Espanha) sobre o reality show espanhol Los Gypsy Kings. Será feita uma breve discussão teórica sobre a representação da população cigana nos meios de comunicação espanhóis. Para compreender o debate, mostraremos dados sobre seu consumo televisivo, focando nos programas de entretenimento, com informações sobre preferências de canais de televisão e tipos de programa. Em seguida, apresentaremos as reflexões da mostra sobre o reality show. A metodologia se baseia em entrevistas semiestruturadas com 20 ciganos e ciganas, realizadas entre os anos de 2017 e 2019. Os resultados mostram as semelhanças na representação das populações ciganas em conteúdos de entretenimento e informativos. Vemos também que o consumo de reality shows e o debate público proposto por associações ciganas sobre Los Gypsy Kings estão refletidos nas reflexões da mostra.

Palavras chave: População Cigana. Consumo Televisivo. Representação. Los Gypsy Kings. Reality show.

\section{Introducción}

Teniendo en cuenta la programación televisiva española de los últimos años, proponemos en este artículo exponer parte de los resultados de una investigación sobre el consumo mediático y cultural de la población gitana de Barcelona. Como parte de un trabajo más amplio, nos enfocaremos aquí en el consumo televisivo de este grupo social, exponiendo las preferencias y los usos de este medio de comunicación, especialmente aquellos relacionados a los programas de entretenimiento. Se verá cómo los canales españoles privados generalistas tienen una gran penetración en la audiencia gitana que formó parte de la investigación y qué posición ocupan los reality shows en este consumo.

Estas informaciones nos interesan en la medida que nos ayudan a comprender cómo este consumo influye en las percepciones que la población gitana tiene sobre el reality show Los Gypsy Kings ${ }^{2}$, que desde 2015 sigue la vida de algunas familias gitanas españolas. El objetivo es comprender la opinión de la muestra sobre dicho programa y en qué medida producciones como esta afectan su día a día. Además, nos interesa saber si las denuncias

2 El debate sobre la estructura y el contenido de Los Gypsy Kings puede ser encontrado en Segarra (2018). 
y movilizaciones de asociaciones gitanas en contra el reality show están presentes en los discursos de las muestras.

Es importante destacar que las reflexiones sobre este programa deben ser leídas a partir de lo que implican los reality shows en la programación televisiva y también a partir del contexto de invisibilización de la comunidad gitana en los medios de comunicación españoles. Este tema se muestra relevante debido al hecho de que Los Gypsy Kings llegó a su sexta temporada en 2020. Además, destacamos el alcance que tienen los reality shows en la audiencia televisiva, ya que cuando tienen éxito, estos programas atraen tanto o más interés que los programas de la televisión de pago, mostrando así su importancia para la televisión en abierto en la disputa por la audiencia (JENKINS, 2008).

Según Segarra (2018, p. 45), los reality shows “fabrican celebridades” favoreciendo dos perspectivas diferentes y aparentemente opuestas: aquella que es idealizante y empática o la irónica y burlona que es “incluso a veces agresivamente humillante”. En esta segunda perspectiva, el espectador es puesto en una situación de superioridad en relación a estas “celebridades”. Además, este tipo de programa "proporciona a los consumidores un flujo estable de dramas éticos” que ordenan las discusiones de la audiencia también sobre aspectos morales, reforzando o cuestionando algunos valores. Los participantes del programa "se exponen a un juicio del público” mientras “el público reitera sus propios valores” (JENKINS, 2008, p. 123).

Esta exposición suele venir acompañada de una demanda por la autenticidad de los participantes del reality show (CAMPANELLA, 2013; COULDRY, 2008), que juegan la paradoja de ser auténticos en un contexto de performatividad. Al cumplir esta exigencia, cada vez más presente en la audiencia, los participantes de los reality shows garantizan su espacio en estos programas a la vez que consiguen consolidar su rol de “celebridad”. En el caso de Los Gypsy Kings, a pesar de no ser un reality de competición, es cierto que el carisma de las familias participantes junto a la audiencia puede influir en la decisión de mantenerlas o no en las temporadas siguientes.

Aunque el reality show sea definido como un género que exhibe y explora la dimensión espectacular de la vida cotidiana de los participantes, que son expuestas representando a si mismas en diferentes situaciones, es importante destacar que tales situaciones son producidas por el equipo del programa, bajo un guión, reglas de funcionamiento y una lógica propia de la producción televisiva y de los formatos ficticios (FRANÇA; SOUZA, 2016).

Además, considerando las reflexiones de França y Souza (2016), esta representación de si mismo puede resultar en la escenificación de roles y en la utilización de modelos sociales aceptados o esperados por la audiencia, dialogando así con los estereotipos presentes en la sociedad. Así, podemos "analizar la propia propuesta de programa en cuanto a una estructuración de sentido que se utiliza de modelos narrativos corrientes y [que] resuena cuestiones sentidas y vividas socialmente” (FRANÇA; SOUZA, 2016, p. 181).

Estas características del reality show nos lleva a enfocar este texto no sólo en el consumo de este programa, sino también en las reflexiones acerca de la representación 
mediática de la población gitana en España, especialmente aquella producida por los medios informativos. Creemos que los contenidos de entretenimiento reproducen los enfoques dados por la prensa en su abordaje de este grupo minoritario, considerando que ella "ha sido clave en la difusión de la imagen socio-cultural de los gitanos” en España (OLEAQUE, 2014, p. 3). Para este debate, destacamos las contribuciones de Willem (2010) y Oleaque (2014).

Según Segarra (2018, p. 41), "igual que todos los individuos racializados en una España que aún se percibe blanca”, la población gitana no está presente en la televisión generalista del país. Cuando esta realidad cambia, ello se da por medio de programas como “Palabra de Gitano” y Los Gypsy Kings que dan la falsa sensación de que se está dando espacio a estos grupos, cuando en realidad se sigue proyectando una imagen estereotipada.

\section{La representación mediática de las poblaciones gitanas}

Los medios tienen un rol importante a la hora de modelar los sujetos sociales, ya que fijan significados sobre aquello que es representado. Según Willem (2010, p. 25), “los mass media son instituciones especializadas en la producción de significados a través de representaciones” y ello no lleva solamente a la creación de estereotipos y diversos tipos de discriminación, sino que también interfiere en la manera en que los propios grupos representados se ven a si mismos, haciendo que muchas veces pasen a comportarse de la manera que se les representan (WILLEM, 2010), respondiendo a lo que creen que se espera de ellos.

Este es el caso de programas de entretenimiento como "Palabra de Gitano"3 que, según Oleaque (2014), tuvo un rol importante en la interiorización o refuerzo de estereotipos entre la población gitana de España. Según este mismo autor (2014, p. 64), el programa, por un lado, "ha triunfado entre los gitanos más marginales - a los que entroniza como representantes de lo gitano” y, por otro lado, ha sido muy criticado por los miembros de la comunidad que se sentían ridiculizados.

Destacamos que, aunque este proceso de interiorización pueda ocurrir, no debemos olvidarnos que el consumo mediático de minorías étnicas es complejo (TSAGAROUSIANOU, 2001) y por ello "diversos factores contribuyen a que existan diferentes niveles de resistencia a los intentos de estereotipación de los grupos minoritarios” (GONÇALVES, 2020a, p. 171).

Investigaciones sobre la representación de las minorías en diferentes partes del mundo han presentado dos aspectos fundamentales sobre este proceso: "por un lado, la sub-representación de grupos de minorías culturales y, por otro, su representación estereotipada” (WILLEM, 2010, p. 29). Según esta autora, los estereotipos difundidos en los medios de comunicación tienen más fuerza que aquellos que surgen del cotidiano, porque los mensajes son producidos de manera altamente profesional y, en consecuencia,

3 Programa emitido por La Cuatro el 2013 en el formato docu-reality, abordando temas relacionados a la población gitana. 
son estructuralmente más sofisticados. Aunque también debe tenerse en cuenta, evidentemente, su poder como difusores.

En el caso de la población gitana en España, se pueden mencionar muchos trabajos e informes sobre su representación en los medios de comunicación, como es el caso del “Periodistas contra el racismo? La prensa española ante el Pueblo Gitano”, producido anualmente por la Unión Romaní desde 1995. Ya Oleaque (2014), en su tesis doctoral, recoge también estudios anteriores a su trabajo, cuyos análisis pueden resumirse en dos ideas: la recurrente presencia de estereotipos basados en el folclore y una relación directa del colectivo con situaciones de conflicto.

En su tesis doctoral, Oleaque (2014) destaca que los individuos retratados en los textos periodísticos analizados son siempre leídos a partir del grupo, de una colectivización acompañada de la despersonalización. La palabra que más veces aparece relacionada con gitano/a/os/as es "familias”, seguida de otras que también hacen referencia a un grupo como “clan”, “etnia” y “raza”, por ejemplo. Esto se debe a que gran parte del material analizado está relacionado con situaciones de conflicto y, en ese contexto, "los gitanos” son vistos como colectivo. Sin embargo, cuando se trata de logros, talento o conquistas, se destaca al individuo, sea como excepción o desde una perspectiva folclorizada.

En los textos que tienen un abordaje más folclórico, los gitanos tienen un papel activo como responsables de su propio éxito y, en cambio, en las materias en que están ligados a “problemas”, aunque también pueden aparecer como responsables de los "problemas” que afectan a la sociedad mayoritaria, suelen tener un papel pasivo al ser presentados como pretexto para cuestionar decisiones y acciones del gobierno, sean en ámbito local, nacional o, incluso, internacional. Cuando se trata del Pueblo Gitano, las conductas de desviación son atribuidas al grupo étnico por la sociedad mayoritaria. Mientras que cuando estas conductas son encontradas en los no gitanos, se responsabiliza al individuo y no a un colectivo (GARRIGA apud. AYUNTAMIENTO, 2015).

Así, no es de extrañar que el espacio dado a los gitanos para que expongan su punto de vista sobre los hechos sea casi nulo o no alcance a ayudar a comprender la complejidad del contexto. Según Oleaque, son referencias que ridiculizan, vulgarizan o refuerzan experiencias marginalizadas: "Cuando se utiliza, no ayuda a tener una imagen más profunda y equilibrada de 'los gitanos’ como grupo, sino a transmitir información elemental que encaja con estereotipos en forma de lamento y queja, o de ripios raciales” (OLEAQUE, 2014, p. 288).

En resumen, la mayoría de los textos destacan el colectivo gitano desde la perspectiva del peligro o amenaza frente a la sociedad mayoritaria, justificando así determinadas acciones hacia el grupo. En segundo lugar, son destacados desde la cultura, sea en aquellos textos que hablan de la música, sea en los que relacionan sus condiciones sociales con determinados rasgos culturales. Por último, en ninguno de los medios se reconoce la existencia del racismo hacia el colectivo gitano en España.

Sin embargo, aunque estas representaciones estén presentes en los medios de comunicación tradicionales, es cierto que las “audiencias pertenecientes a minorías étnicas” 
también reivindican “inclusión en el 'mainstream”” (ROSS; PLAIDON, 2001, p. XIV), ya que las “normas culturales y comportamientos” producidos o reforzados por estos medios influyen "en la manera como la sociedad mayoritaria se relaciona con las minorías en su cotidiano” (ROSS; PLAYDON, 2001).

\section{Metodología}

De carácter cualitativo, la metodología para la producción de los datos presentados en este artículo se basa en trabajo de campo que incluye la observación participante y la realización de entrevistas semiestructuradas entre los años de 2017 y 2019, en la provincia de Barcelona, en Cataluña. Creemos que esta metodología “contribuye con la construcción de un hacer científico más horizontal, especialmente en las investigaciones con grupos sociales históricamente silenciados dentro de las estructuras de poder del saber científico” (GONÇALVES, 2020b, p. 46-47). La elección por una metodología cualitativa se debió a las posibilidades que ella proporcionó para el desarrollo de esta investigación: el conocimiento profundizado del contexto social e histórico de esta población, la interacción, el diálogo, el afecto y la construcción de redes y, finalmente, el compromiso de la investigadora con el propio proceso metodológico (GONÇALVES, 2020b) ${ }^{4}$.

Las entrevistas fueron realizadas con 20 personas, siendo 10 hombres y 10 mujeres, con edad entre 18 y 47 años, con diferentes niveles de estudio, profesiones y de diversos barrios y ciudades de la provincia. Las entrevistas duraron una media de 40 minutos. La muestra será identificada aquí por un número de 1 a 20, siendo el número 1 el más joven y por la letra $\mathrm{H}$ para hombres y $\mathrm{M}$ para mujeres.

En lo referente a este artículo, los datos presentados se refieren a las cuestiones puestas a la muestra relacionadas a dos puntos: su consumo televisivo y el consumo de contenidos sobre la población gitana. En el primer caso, los y las entrevistadas contestaron a la pregunta sobre qué suelen ver en la tele. Las respuestas incluían tanto cadenas de televisión en general como programas específicos. Luego se dividió los datos entre noticias y entretenimiento, siendo este último el foco de este trabajo. Las respuestas de la muestra nos guiaron para la organización de los programas mencionados en diferentes géneros: Infantil, Ficción, Información ${ }^{5}$, Reality, Humor, Infoshow y Deporte. Aparte de las menciones a preferencias por determinados tipos de contenido, también se les fue preguntado con qué frecuencia suelen mirar la televisión y en qué circunstancia.

Ya en lo referente al consumo de contenidos sobre la población gitana, primeramente, se les fue preguntado si conocían contenidos de este tipo y, luego, si solían seguirlos. Los dos tipos de contenidos mencionados fueron las noticias y algunos contenidos de

4 Para un debate más profundizado sobre la metodología ver "Pesquisa participante e comunicação dialógica: a experiência com as comunidades ciganas da Espanha" (GONÇALVES, 2020b).

5 Aparte de las noticias, en lo referente al entretenimiento hemos definido como Información programas como documentales, de investigación, debates o entrevistas. 
entretenimiento, habiendo una mención mayoritaria al docu-reality "Palabra de Gitano" y al reality show Los Gypsy Kings. La pregunta siguiente se refería entonces a la opinión de la muestra sobre estos programas.

Recogeremos aquí aquellas referentes específicamente a Los Gypsy Kings por haber ganado más destaque en los discursos de la muestra, considerando que durante el trabajo de campo el reality seguía siendo emitido, mientras "Palabra de Gitano” ya no formaba parte de la oferta televisiva.

A partir de la sistematización y organización de los discursos recogidos, se procedió al análisis enfocándonos en todo lo referente a las preferencias, a las prácticas y a las opiniones de la muestra en relación a estos dos aspectos mencionados anteriormente, preocupándonos siempre por no perder la coherencia de sus discursos, ya que la entrevista semiestructurada tiene como característica respuestas abiertas.

\section{Análisis - el consumo televisivo}

Un consumo frecuente de televisión aparece en más de la mitad de la muestra consultada para esta investigación, habiendo más seguimiento entre los adultos, pero todos ellos afirmaron verla en algún momento. El consumo más frecuente se vincula sobre todo con el seguimiento de noticias, mientras que el consumo esporádico es más mencionado en relación con el entretenimiento.

Con una oferta de 32 canales $^{6}$, la televisión generalista y estatal es la más citada, siendo TV3 la única autonómica que aparece en el listado, de un total de 8 canales disponibles en Cataluña. Ninguna emisora local es mencionada, a pesar de la oferta de 51 canales de este tipo, mientras que la única cadena global citada más de una vez (dos veces en total) es HBO. Telecinco y Antena 3 son las más vistas, seguidas de Cuatro, TVE, Sexta y TV3. Entre los canales más citados están dos públicos: TVE, en ámbito estatal, y TV3, autonómica de Cataluña. Si miramos la preferencia por sector industrial, Mediaset, cuyo público mayoritario es más bien de jóvenes y hombres (SEGARRA, 2018), aparece en primer lugar, seguida de Atresmedia Televisión.

La mitad de la muestra tiene la costumbre de seguir la programación televisiva de entretenimiento. Más mujeres que hombres afirmaron no ver la televisión con mucha frecuencia con este fin, sea por falta de tiempo (4), sea por falta de interés (2). Ser madre fue mencionado por dos entrevistadas como el motivo de su falta de tiempo, aunque las dos igualmente indicaron que antes de que nacieran sus hijos tampoco veían mucho la televisión. Otra de las interlocutoras explicó que cuando era más joven sí que se ocupaba más de este medio, especialmente viendo las telenovelas, y que ahora no lo hace porque cuando tiene tiempo de dedicarse a ello no es el horario de emisión de los contenidos que le gusta acompañar.

6 Los datos sobre la oferta de canales son del Boletín de Información sobre el Audiovisual en Cataluña del 2018, producido por el Consejo del Audiovisual de Cataluña. 
Entre los hombres, tres afirmaron no verla por falta de tiempo y dos porque la televisión les aburre. Para uno de ellos, la televisión es un medio ya muy antiguo, como un aparato que le sirve más para jugar a la consola o visionar vídeos que tiene en el móvil. Otro afirmó que antes de casarse solía ver más la televisión, especialmente películas por la noche.

Entre aquellos que presentan menor interés por la programación televisiva, muchos apuntan el estilo de vida como justificativa, como las responsabilidades, los hobbies y los hijos. Además, dos entrevistados mencionaron su preferencia por Netflix en lugar de la programación televisiva lineal tradicional, siendo que uno de ellos apuntó también la publicidad $^{7}$ de la televisión como una razón para preferir la plataforma online de reproducción de vídeos.

Una de las entrevistadas tiene una visión muy crítica sobre la programación televisiva, explicando por qué en su opinión la gente, incluyendo niños y niñas, acompaña lo que ella considera telebasura:

(...) es que está al orden del día, en cualquier canal tienes telebasura. Pues eso es lo que consume la gente, a no ser por personas también que estén muy ocupadas, que por su ritmo de vida y tal (...) la mayoría consume lo mismo, pero es lo que en el momento está saliendo. Digamos que somos mucho de actualidad, de lo que actualmente hay, pues también nosotros somos permeables y también pues eso nos cala y nos acaba haciendo víctima del consumismo de lo que los medios ofrecen (E17M).

Entre las mujeres, 8 de las entrevistadas aprovechan para ver la televisión cuando están con algún familiar: hijos, parejas o padres. Ello se da también como una estrategia para compartir el momento con estos familiares. Algunas veces, la programación seguida en estos momentos se corresponde menos con sus gustos personales que con las preferencias de sus familiares, lo que nos muestra que la dieta televisiva de la muestra puede estar determinada también por la compañía a la hora de ver la televisión y no solamente por intereses individuales.

En relación a las preferencias de entretenimiento, la mayoría afirma ver Ficción (14) - siendo películas y series las más mencionadas -, seguido de Reality (6), Infantil (5) e Información (4). Si separamos por sexo, la Ficción sigue siendo la preferencia de ambos, mientras el Reality es una unanimidad entre las mujeres adultas y se destaca entre los hombres jóvenes.

7 Según el último Boletín de Información sobre el Audiovisual de Cataluña, producido por el Consejo de Audiovisual de Cataluña, la Directiva de Servicios de Comunicación Audiovisual de la Unión Europea publicada en noviembre de 2018 ha flexibilizado la regulación de publicidad en la televisión lineal, permitiendo que los prestadores la ubiquen hasta un $20 \%$ del tiempo de emisión en las franjas horarias de $6 \mathrm{~h}$ a $18 \mathrm{~h}$ y de $18 \mathrm{~h}$ a $24 \mathrm{~h}$. Antes los prestadores tenían un máximo de 12 minutos por hora natural. 


\section{El programa Los Gypsy Kings}

Considerando la gran penetración que la televisión sigue teniendo en la audiencia gitana y que el reality show es el segundo género de entretenimiento más seguido, pasamos ahora a discutir el caso del programa Los Gypsy Kings. El reality show ha sido mencionado por 16 de los entrevistados cuando fueron preguntados sobre productos mediáticos y culturales, producidos sobre gitanos. El programa es descrito por su productora (La Competencia) como un docu-reality de humor, mientras Segarra (2018) lo califica como un reality show. Según la autora, Mediaset, grupo de comunicación responsable por emitir el programa en La Cuatro y tercera emisora más vista por nuestra muestra, se destaca en este género.

Volviendo a la relación que los reality’s crean entre participantes y público, Segarra (2018, p. 45) destaca que en el caso de Los Gypsy Kings, dos perspectivas son combinadas. De un lado, "el tratamiento de los personajes es empático y horizontal ya que se trata de cuestiones 'generales' con las que todo el público puede identificarse” y, de otro, es “irónica y vertical - especialmente cuando el guión apunta a la incultura e, incluso, la ignorancia de los personajes a veces presentada como abismal”. Mientras la primera perspectiva atrae la atención del público, sea gitano o no, la segunda contribuye con la estigmatización de la población gitana. Por eso, no es difícil encontrar opiniones contrarias al programa en nuestra muestra.

En estos casos, los entrevistados argumentan que las familias retratadas allí no representan la realidad de la mayoría de los gitanos del país. Sin embargo, al identificar todos los personajes del programa como gitanos, se presupone que "todos pertenecen a un grupo más o menos homogéneo” (SEGARRA, 2018, p. 45). Como explica E11M: “por salir en la tele como que nos representan un poco a todos. Y claro, están manchando la imagen muchísimo, porque para nada los gitanos son así. ¿Que hay? Sí que hay, pero no todos”.

Así mismo, aunque se les presente como pertenecientes a grupos económicos diferentes, “todos sufren de una profunda incultura”, siendo su capital cultural presentado como abajo de la media española (SEGARRA, 2018, p. 46). Ello contribuye en que la sociedad mayoritaria construya o refuerce una imagen distorsionada sobre la población gitana.

(...) odio este programa porque no es la realidad del Pueblo Gitano, entonces esto crea que la gente no gitana se cree una realidad que es completamente incierta a la que es (...) Claro que estos programas quieren el folclore para que la gente pues vea. (...) Yo como mujer gitana me siento indignada cuando veo (E18M).

(...) cuando entré a estudiar dijeron: pero si las niñas gitanas lo que hacen es casarse como la Rebe de Plasencia, sólo hacen que ronear y casarse como los de Gypsy Kings o me han preguntado de qué viven los gitanos porque ahí sale una pareja y sólo se dedican al mercado, pero sin embargo tienen dinero para hacer cumpleaños como si fueran bodas y de viajar y de tener lujos como 
si fueran millonarios. Y la gente se pregunta de qué viven los gitanos y qué dinero sacan del mercado para tener la vida que se muestra en Los Gypsy Kings. Pero no es real. Una persona, unos gitanos que trabajan en el mercado tienen a duras penas para comer y poco más (E11M).

(...) a mí me da mucha rabia ese programa y no lo veo. (...) Porque dejan los gitanos con una mala imagen, (...) no digo que sean malas personas ni que nada, pero que sacan sólo como su parte de humor, y más, poco inteligente por llamarlo bien $(\mathrm{E} 1 \mathrm{H})$.

(...) lo malo es que la sociedad se queda con esta imagen y creen que esa es la vida del gitano y que el gitano vive de esa forma. Entonces no me gusta por eso porque están dando una imagen que la sociedad está recogiendo errónea que no tiene nada que ver $(\mathrm{E} 4 \mathrm{H})$.

Además, para dos de los entrevistados, programas como este pueden contribuir a que vecinos cambien la buena percepción que tienen sobre ellos, rompiendo con lo que la comunidad gitana ha estado construyendo de cara a la convivencia con la sociedad mayoritaria:

A mí me ha pasado con mi vecina, cuando empezaron estos programas, me dice: ah, pero ¿vosotros también hacéis esto? Y le dije: ostras, nos conoce de toda la vida, desde el año 75 que viven aquí, que sois vecinas. Digo, y ahora te piensas que nosotros somos así. Cuando nos conocemos mucho. O sea, yo he comido en tu casa, tu hijo ha comido en mi casa, nos hemos criado juntos, hemos celebrado San Juan juntos y se piensan que lo que ven en la tele, como la mayoría de la gente, es verdad. Entonces claro, cambia mucho lo que hemos conseguido con la convivencia que un programa rompa todo esto (E12M).

(...) luego yo bajo aquí a tomar café y me dicen: oye, y ¿esto que hacen ellos en la tele lo hacéis vosotros? Yo no me gasto no sé cuantos mil euros en una boda, primero porque no sé de dónde voy a sacarlo (E6H).

Por otro lado, al mismo tiempo que hay la reflexión sobre cómo este programa perjudica la representación de la población gitana, también se reconoce que en este caso hay una quiebra del principio de autenticidad de los participantes del reality, dejando más evidente su carácter ficcional.

(...) no deja de ser un reality show que son mandados a hacer lo que ellos quieren y son como actores que hacen lo que les dicen [...] Yo entiendo que ellos lo hacen porque es una oportunidad que ha salido para ganar dinero o para lo que sea. Más que a los gitanos que son los protagonistas, le echaré la 
culpa a los productores que manipulan y se aprovechan de ciertas familias para beneficiarse ellos (E4H).

(...) la gente lo sabe que ellos cumplen un papel, que tienen unas órdenes, que reciben un dinero por lo que están haciendo y que está todo súper pactado (E12M).

(...) esta gente lo hace todo por dinero, básicamente hacen por dinero porque es muy evidente, hacen increíblemente [...] una familia gitana entera se pone a hacer el tonto delante de una cámara porque están dando tanto dinero y no solamente se están perjudicando ellos, sino que me están perjudicando a mí directamente, no indirectamente, directamente (E6H).

Una de las entrevistadas lo sitúa al nivel de otros reality shows como, por ejemplo, “Mujeres y hombres y viceversa” o "Granjero busca esposa"8. Por dos veces, en actividades del asociacionismo gitano durante el trabajo de campo, esta misma entrevistada al referirse a Los Gypsy Kings preguntó por qué los gitanos no pueden tener sus propios friquis (término definido por la Real Academia Española como “persona extravagante, rara o excéntrica”), así como la sociedad mayoritaria tiene los suyos que participan en otros programas.

Su opinión va de encuentro a lo que Ross y Playdon (2001, p. 14) explican al decir que "audiencias minoritarias no quieren favores especiales en términos de representación, sino que más bien les gustaría ver sus muchos y diversos selves y sus diferentes vidas representadas con más equilibrio". Esto es lo que reivindica también E1H, al decir que "hay muchos más gitanos en el mundo para sacar sólo esa clase de personas porque si encima los medios de comunicación no dejan muy buena imagen de los gitanos, pues si ponen un programa así pues nada, más leña al fuego y no me gusta”. Otro entrevistado comparte esta reflexión: "La sociedad a veces desconoce mucho lo que es la figura del gitano, entonces si esa figura estuviese bien marcada y sabrían lo que es, no me importaría que saliese en un reality show...” (E4H).

Algunos de los entrevistados dicen ver el programa, aunque expliquen no sentirse representados por él. Para ellos, se trata de un programa más de entretenimiento que les divierte:

(...) yo no lo veo, pero tengo que decir que en mi casa se ve (...) ellos dicen que para ellos es un programa de entretenimiento y de risa, lo ven como un programa de humor, que se sabe el público [...] Ellos dicen a mí me hace gracia, reírme, como si estuviera viendo yo que sé Los Morancos, pues estoy viendo esto (E12M).

8 “Mujeres y Hombres y viceversa” es un programa de citas que estrenó en 2018, mientras “Granjero busca esposa”, que estrenó en 2008, es un reality show, en el cual 10 granjeros tienen que elegir a su esposa después de conocer y convivir con mujeres de la ciudad. 
Yo, Los Gypsy Kings, la verdad que no suelo ver, no me gusta. Una cosa que, si lo veo, se pone en casa así y se ve un poquito, por reírte también, a veces lo ves por reírte, pero no me gusta, no me hace gracia (E2M).

Es verdad que si te digo que no lo veo es mentira, porque sabes lo que hay lo ves y muchas veces hasta me río, pero luego te paras a pensar fríamente y te pones enfermo (E6H).

Sí, hay cosas que no estoy de acuerdo, en algunos comentarios que hacen ellos, la vida que tienen ellos, alguna cosa, pero sí, lo suelo ver. (...) Sí, sí, me río. (...) Bueno la vida que tienen los de la tele no la tenemos nosotros. Pero muchas cosas sí. Muchas opiniones que tienen ellos sí. (...) A veces se mofan de nosotros. Porque empiezan a hablar a lo mejor de una manera que no la hablamos todos (...) Y a lo mejor no somos así, la mayoría no somos así... (E13H).

Otros entrevistados que trabajan en el mundo asociativo explicaron haber visto el programa por lo menos una vez para poder construir su propia opinión acerca del mismo. Es importante destacar que, a cada nueva temporada del programa, asociaciones de toda España difundieron notas de repudio o, incluso, presentaron denuncias sobre el modo como los gitanos son retratados en el reality show. Según estas notas, el programa incide "en una imagen estereotipada y caricaturizada de la comunidad gitana”, además "se trata de una mirada vertical, alejada y autoritaria cuyo objetivo es la producción de beneficios económicos, sin más.” ${ }^{9}$ Esta posición coincide, por un lado, con la percepción de parte de la muestra al mismo tiempo que influye en la formación de su opinión, ya que hay un contacto próximo con las diferentes entidades y asociaciones del país, especialmente por medio de las redes sociales.

\section{Conclusiones}

A pesar de la representación estereotipada dada a la población gitana por los medios de comunicación españoles, hemos visto que hay un consumo significativo de la oferta televisiva, que aún tiene una gran penetración en el público gitano, aunque sea menor entre los más jóvenes, con especial atención a las cadenas privadas españolas y generalistas como Telecinco, Antena 3 y La Cuatro. Los programas de entretenimiento atraen menos interés que las noticias, pero aun así alcanza la mitad de la muestra que tiene preferencia por ficción y reality shows.

En relación al programa Los Gypsy Kings, hemos visto que hay, entre los entrevistados, una postura crítica cuanto a la manera como su pueblo es reducido a la representación de

9 Artículos y comunicados producidos por entidades gitanas sobre el programa. Disponible en: https://www.gitanos.org/actualidad/ dossieres/115502.html.es. Acceso en: 4 mar. 2019. 
algunas familias. Por otro lado, parte de la muestra también admite que se divierte con el programa, aunque no se sienta representada por él. Estas dos perspectivas nos remiten a otros estudios de audiencias de minorías racializadas, que al no reconocerse en la única identidad que les es ofrecida en los medios de comunicación, rechazan dichas representaciones "con humor y rabia” (ROSS; PLAYDON, 2001, p. XVI-XVII). Además, el hecho de que los reality shows están entre los programas preferidos de la muestra nos lleva a creer que hay una familiaridad con su formato y su lenguaje, lo que influye en el modo como los y las interlocutoras evaluaron el programa Los Gypsy Kings.

En lo referente a la relación de la representación de Los Gypsy Kings con los enfoques dados por los contenidos que salen en la prensa, hay la coincidencia en el abordaje folclórico de familias gitanas y de homogeneidad en la cual las poblaciones gitanas son retratadas desde la colectivización. La ridiculización y vulgarización apuntadas por Oleaque (2014) se notan en las reflexiones de la muestra al hablar de un humor poco inteligente, relacionado a una incultura, que es atribuido a los personajes del reality show. Esta incultura refuerza el estereotipo creado también por la prensa en la que las condiciones sociales de la población gitana es consecuencia de sus rasgos étnicos. Aunque no aparezca en Los Gypsy Kings la relación directa entre gitanos y conflicto, sí que es cierto que, según uno de los entrevistados, se puso en cuestión el origen del dinero de una de las familias retratadas en el programa.

Al denunciar el reality Los Gypsy Kings, las entidades, asociaciones y activistas gitanas cuestionan más que un género televisivo, cuestionan el hecho de que este colectivo no tiene su derecho a una representación que enseñe otras identidades gitanas, que sean múltiples y diversas, presente en los medios de comunicación del país.

Como ya hemos discutido anteriormente, los medios de comunicación tienen más fuerza en la difusión de estereotipos por la profesionalidad en la producción de sus mensajes (WILLEM, 2010), por ello, como apunta Segarra (2018, p. 47), el guión seguido por los personajes en Los Gypsy Kings tiene un poder de legitimación diferente de los informativos, ya que "estas emisiones también fabrican la realidad, especialmente en lo referente a las relaciones sociales, entre 'razas' o 'culturas' diferentes...”.

Igual que no se puede hablar de la historia de España sin hablar de su población gitana, no se puede hacer un debate serio sobre la televisión española sin cuestionar sus contenidos, en lo referente a las representaciones presentes o ausentes de la minoría étnica más antigua del país y como ello contribuye al "racismo ordinario" (SEGARRA, 2018) vivido por esta población.

\section{Referencias}

AYUNTAMIENTO DE BARCELONA. Estratègia local amb el poble gitano de Barcelona: diagnosi i línies d'actuació, 2015. Disponible en: https://www.encurtador.com.br/yGTV8. Acceso en: 29 dic. 2020.

CAMPANELLA, B. Tirando as máscaras: o reality show e a busca pela autenticidade no mundo contemporâneo. Revista da Associação Nacional dos Programas de Pós-Graduação em Comunicação E-compós, Brasília, v. 16, n. 1, p. 1-17, ene./abr. 2013. 
COULDRY, N. Reality TV, or the secret theater of neoliberalism. Review of Education, Pedagogy and Cultural Studies, v. 30, n. 3, p. 3-13, 2008. DOI: 10.1080/10714410701821255.

FRANÇA, V.; SOUZA, F. 'Troca de Família': espiando as famílias, enxergando a sociedade. Intercom: Revista Brasileira de Ciências da Comunicação. São Paulo, v. 39, n. 1, p. 177-192, ene./abr. 2016. Disponible em: https://doi.org/10.1590/1809-58442016110. Acceso en: 29 dic. 2020.

GONÇALVES, G. M. Pesquisa participante e comunicação dialógica: a experiência com as comunidades ciganas da Espanha. Revista de Comunicação Dialógica, n. 3, año 2, p. 34-50, 2020a. Disponible em: https://doi.org/10.12957/rcd.2020.50867. Acceso en: 29 dic. 2020.

GONÇALVES, G. M. Pueblo gitano y consumo mediático informativo: ¿audiencia crítica y activa? RAEIC: Revista de la Asociación Española de Investigación de la Comunicación, v. 7, n. 13, p. 164-184, 2020b. Disponible en: https://doi.org/10.24137/raeic.7.13.8. Acceso en: 29 dic. 2020.

HUERTAS, A. Yo soy audiencia - Ciudadanía, público y mercado. Barcelona: UOCpress, 2015.

JENKINS, H. Cultura da convergência. São Paulo: Aleph, 2008.

OLEAQUE, J. Los gitanos en la prensa española - Variación y reiteración de los planteamientos de los diarios ABC, El País y La Vanguardia en la representación de los gitanos como grupo (1981-2010). Tesis. Universitat de València: Valencia, España, 2014. Disponible en: http://hdl.handle.net/10550/42269. Acceso en: 29 dic. 2020.

ROSS, K.; PLAYDON, P. (ed.). Black Marks: Minority Ethnic Audiences and Media. Hampshire: Ashgate Publicshing Limited, 2001.

SEGARRA, M. Entre voyeurisme et ironie: représentations télévisuelles des Gitanxs. Sociétés \& Représentations, v. 45, n. 1, p. 41-52, 2018. Disponible en: https://oi.org/10.3917/sr.045.0041. Acceso en: 29 dic. 2020.

TSAGAROUSIANOU, R. Ethnic minority media audiences, community and identity:the case of London's South Asian and Greek-Cypriot communities. In: ROSS, K.; PLAYDON, P. (ed.). Black Marks: Minority Ethnic Audiences and Media. Hampshire: Ashgate Publicshing Limited, 2001.

WILLEM, C. 'Roots and Routes' - Young people from diverse ethno-cultural backgrounds constructing their identities using digital media. Tesis (Departamento de Didáctica de la Educación Visual y Plástica). Universitat de Barcelona: Barcelona, España, 2010. Disponible en: https://www.encurtador.com.br/iCJNZ. Acceso en: 29 dic. 2020.

\section{Gabriela Marques Gonçalves}

Miembro externo del Instituto de la Comunicación (InCom-UAB), doctora en Comunicación Audiovisual y Publicidad por la Universidad Autónoma de Barcelona (UAB-España). Tiene Máster en Comunicación por la Universidade Federal de Juiz de Fora (UFJF-Brasil). Se dedica a temas relacionados a los Estudios Gitanos, racismo y comunicación. E-mail: gabimarques@pm.me.

Recibido en: 02.06.2020

Aceptado en: 11.02.2021

Este artículo está publicado en acceso abierto (Open Access) bajo la licencia Creative Commons Attribution Non-Commercial (CCBY-NC), que permite el uso, distribución y reproducción in cualquier medio, sin restricciones, desde que sin fines comerciales y que el trabajo original sea correctamente citado. 